\title{
Radiological examinations of the anatomy of the inferior turbinate using Digital Volume Tomography (DVT)
}

\author{
L. Balbach ${ }^{1}$, V. Trinkel ${ }^{1}$, C. Güldner ${ }^{1}$, S. Bien ${ }^{2}$, A. Teymoortash ${ }^{1}$, J.A. Werner ${ }^{1}$, \\ M. Bremke ${ }^{3}$
}

\author{
Department of Otorhinolaryngology, Head and Neck Surgery, UKGM, Marburg, Germany \\ Department of Neuroradiology, UKGM, Marburg, Germany \\ Department of Otorhinolaryngology, Head and Neck Surgery, University Hospital, Cologne, Germany
}

SUMMARY Background: Since the last 120 years there were only few descriptions of the anatomical sizes of the inferior turbinate in the literature. On this background the current study should evaluate the radiological dimensions of the inferior turbinate and the septum using DVT. Methods: The latest generation of the Accu-I-tomo was used. The data of 100 adult patients have been evaluated. Results: The bony length was found to be $38.9 \mathrm{~mm}$, the mucosal length $51.0 \mathrm{~mm}$. The findings of the total mucosal thickness at different measuring points were between $8.1 \mathrm{~mm}$ and $10.9 \mathrm{~mm}$, those of the bony thickness were between $0.9 \mathrm{~mm}$ and $2.3 \mathrm{~mm}$ and those of the bony height were between $3.9 \mathrm{~mm}$ and $20.8 \mathrm{~mm}$. Conclusion: The results of this radiological study are able to point out the importance of preoperative anatomical evaluation of radiological images. The preoperative focus on the individual anatomy is very important because of the choice of an adequate surgical treatment. Today new radiological techniques can help to find out whether the reason for hypertrophied turbinates is caused by bone, mucosa or both. This knowledge enables a concerted treatment concept.

Key words: Digital Volume Tomography (DVT), Cone beam tomography, Rotational tomography, inferior turbinate anatomy, nasal obstruction

\section{INTRODUCTION}

In 1893, Zuckerkandl (1) already described the inferior turbinate in his monograph based on cadaveric preparation as an independent bone. It has its origin from the lateral nasal wall. The main function of the turbinates is warming, cleaning and moisturizing of the inspired air ${ }^{(2)}$. Thereby the inferior turbinate has a central importance because of the high potential to swell and therefore to change its volume. Zuckerkandl (1) stated the length of the mucosa covered inferior turbinate between 25 and $49 \mathrm{~mm}$ and its width between 5 and $16 \mathrm{~mm}$. He subdivided the turbinate in a body and three processes.

In 1981 and 1982, Lang ${ }^{(3,4)}$ also analysed the dimensions of the inferior turbinate in human bodies. In the first study ${ }^{(3)}$ he measured 95 medianly divided halves of heads. In the second

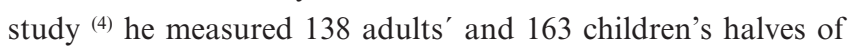
heads.

One of the most common findings in patients suffering from sinunasal pathologies is nasal obstruction. The main causes for this symptom in non-polypoid diseases are septal deviations and hypertrophy of the nasal turbinates. Septal deviation was described in $21 \%$ of female and $72 \%$ of male patients already in 1895 by Stier ${ }^{(5)}$. Gray ${ }^{(6)}$ specified a cartilaginous and a cartilaginous-osseous type of septal deviations with common combinations of both types. In many patients hypertrophy of an inferior turbinate occurs as a kind of compensatory mechanism on the concave side of the deviated septum ${ }^{(7,8)}$. Enlargement of the inferior turbinate can be caused by mucosal ${ }^{(8)}$, mucosalsubmucosal and bony hypertrophy ${ }^{(9,10)}$. Pneumatisation of the inferior turbinate is a rare cause for its enlargement and was first described by Zinreich ${ }^{(1)}$ in 1988, although it is the most frequent anatomical variation of the middle turbinate ${ }^{(12)}$.

The consequence of these anatomical varieties can be nasal obstruction itself or one of the reasons for chronic rhinosinusitis. Like Mirza ${ }^{(13)}$ showed, in some cases these pathologies can lead to obstructive sleep apnea syndrome (OSAS).

To improve the nasal obstruction caused by a hypertrophied inferior turbinate there is a huge variety of surgical techniques described in the literature of the last 150 years, such as radiofrequency, laterofracture or turbinoplasty for example ${ }^{(14,15)}$. A great number of other methods exists in the literature. 
Digital volume tomography (DVT) is an improvement of panoramic tomography used in dentistry. It enables high quality $3 \mathrm{D}$ images of bony structures and is based on the principles of cone beam tomography ${ }^{(16-18)}$. This means that the DVT scanner generates the data during one single rotation around the patient's head in contrast to CT scanners that need several cycles. In cone beam tomography the X-ray-beam extends conically: the top of the cone is the emitter, the base the detector of radiation. During the rotation up to 1000 two-dimensional slices are created that are used by the software as the basis for the calculation of the cylindrical volume. Furthermore it has a lower radiation dose and less artefacts in comparison to most CT scanners. The limited display of soft tissue may be one disadvantage of DVT compared with CT. The slices can be evaluated in three orthogonal plains in frontal, axial and sagittal manner. Measurements of distances can be performed with the standard software in a simple way.

In the meantime DVT has shown its value in the field of ENT. Initially it was used as diagnostic tool in the lateral skull base, as well as in nasal bone fractures and paranasal sinus diagnostics ${ }^{(16-18)}$. In the case of a nasal bone fracture it is reported that the DVT or CT can give more specific pictures compared to conventional radiography especially if it is a discreet fracture with less dislocation ${ }^{(16)}$. It was also possible to analyse the microanatomic structures of the olfactory cleft and the olfactory fossa (17). Since the introduction of the cylindrical volume of $10 \times 10$ $\mathrm{cm}$ the whole paranasal sinus system can be constituted in one DVT scan in most of the cases. This may improve the diagnostic options of paranasal sinus diseases ${ }^{(18)}$. The digital volume tomograph has a low purchase price for the equipment in comparison to a modern high-resolution computed tomograph.

The aim of this study was to analyse the radiological anatomic dimensions of the inferior turbinate in relation to the nasal septum and the nasal cavity using DVT scans of the midface retrospectively to achieve standard values of the sizes of the inferior turbinates in an adult population of 100 individuals.

\section{PATIENTS AND METHODS}

In the current study the latest generation of the Accu-I-tomo (F17, Morita, Kyoto, Japan) was used. During the examination the patient is sitting in an upright position with the head fixed on an adjustable chair (Figure 1). The region of interest (ROI) is marked by target light beams that can be positioned arbitrarily. The emitter-detector-unit roates along the patient's head in about 18 seconds. During this time about 580 single slices of a section width down to $0.08 \mathrm{~mm}$ are performed. The rotation during the examination creates a cylindrical volume up to $17 \mathrm{~cm}$ in diameter and $12 \mathrm{~cm}$ in height. The size of this volume can be chosen in dependence to the diagnostic requirements from $4 \times 4 \mathrm{~cm}$ up to $12 \times 17 \mathrm{~cm}$. In this study, it sufficed to use a cylindrical volume of $10 \times 10 \mathrm{~cm}$ to visualise the nasal cavity and the adjacent paranasal sinuses. The Accu-I-tomo possesses a D-051 x-ray tube made by Toshiba. The tube voltage was set on $80 \mathrm{kV}$, the tube current was $8 \mathrm{~mA}$. The computed tomography dose index (CTDI) given with this setting was 7.6 mGy and was calculated by the DVT device. After calculation and reconstruction with special software (Idixel, Morita, Kyoto, Japan) the slices are visualised on a monitor. The distance measuring tool of the software was used to perform measurements of the bony and mucosal dimensions of the turbinates and the nasal septum.

The data of 100 adult patients ( 50 male patients, mean age 38.6 years, range 19-83 years; 50 female patients, mean age 38.6 years, range 19-83 years) have been evaluated for this study. They have been examined because of suspected chronic rhinosinusitis (74 patients), nasal bone fracture or blow out fracture (26 patients) between March 2008 and March 2009. Patients with polypoid rhinosinusitis and a history of sinus surgery were excluded from this study.

The measurements were accomplished at four points of the anterior-posterior course of the turbinates, the first at the anterior (M1) and the last one at the posterior (M4) osseous border. The other two points were in between dividing the distance in three identical parts (M2+M3) (Figure 2).

The analysis of the DVT scans contained the bony extent in anterior-posterior and cranio-caudal direction, the general width of the turbinates consisting of the bony and mucosal parts (each at the thickest points), the separate mucosal width of the lateral and medial side of the turbinate (Figures 3, 4). The anterior and posterior overlay was calculated (Figure 5). The insertion angle of the inferior turbinate in relation to the lateral nasal wall was also evaluated (Figure 3). Anatomical variations of the turbinates were analysed as well. The results of the measurements were evaluated by Microsoft Excel ${ }^{\circledR}$.

\section{RESULTS}

The bony length of the inferior turbinate in anterior-posterior direction was found to be $38.9 \pm 4.0 \mathrm{~mm}$. The mucosal length was $51.0 \pm 5.1 \mathrm{~mm}$ (Table 1). The anterior mucosal overlay was $4.6 \mathrm{~mm}$, the posterior overlay was $7.6 \mathrm{~mm}$, whereas the turbinates of the male patients were averaged one millimetre longer than the turbinates of the female patients (Table 2). The bony height at the four mentioned points in all patients was 3.9 $\mathrm{mm}$ at M1, $20.8 \mathrm{~mm}$ at M2, $13.7 \mathrm{~mm}$ at M3 and $4.0 \mathrm{~mm}$ at M4 (Table 3). The bony thickness was $0.9 \mathrm{~mm}$ (M1), $2.7 \mathrm{~mm}$ (M2), $2.3 \mathrm{~mm}$ (M3) and $1.1 \mathrm{~mm}$ (M4) also in anterior-posterior direction. There were maximal results up to $6.0 \mathrm{~mm}$ at $\mathrm{M} 2$ and 6.2 $\mathrm{mm}$ at M3. A summary of all data is shown in Table 3.

The caudal mucosal extensions are $4.4 \mathrm{~mm}$ (M1), $3.6 \mathrm{~mm}$ (M2), $3.8 \mathrm{~mm}$ (M3) and $5.8 \mathrm{~mm}$ (M4) (Table 3). The total mucosal thickness was 8.6 (M1), 8.1 (M2), $8.2 \mathrm{~mm}$ (M3) and $10.9 \mathrm{~mm}$ (M4) (Table 3).

The average of the projection angle at $\mathrm{M} 1$ was $57.0^{\circ}$, at $\mathrm{M} 2$ $63.6^{\circ}$, at $\mathrm{M} 383.2^{\circ}$ and at M4 84.2 (Table 3).

A unilateral pneumatisation of the inferior turbinate was found in one female patient. A bilaterally pneumatised inferior 
turbinate was seen in one male patient (Figure 5a, b). Thirtytwo patients (12 female, 20 male) had a septal deviation over $3 \mathrm{~mm}$ from the midline. There were 48 patients (18 female, 30 male) with a septal spur, which was found in $61 \%$ in the last third of the nasal septum.

Table 1. Bony and mucosal length (in $\mathrm{mm}$ ).

\begin{tabular}{cc}
\hline bony & mucosal \\
\hline $38.9 \pm 4.0$ & $51.0 \pm 5.1$ \\
\hline
\end{tabular}

Table 2. Anterior and posterior mucosal overlay (in $\mathrm{mm}$ ).

\begin{tabular}{cc}
\hline anterior & posterior \\
\hline $4.6 \pm 2.0$ & $7.6 \pm 2.9$ \\
\hline
\end{tabular}

Table 3. Average values (in $\mathrm{mm}$ )

\begin{tabular}{lcccc}
\hline & M1 & M2 & M3 & M4 \\
\cline { 2 - 5 } bony height & $3.9 \pm 1.5$ & $20.8 \pm 5.2$ & $13.7 \pm 3.2$ & $4.0 \pm 2.0$ \\
bony thickness & $0.9 \pm 0.4$ & $2.7 \pm 1.1$ & $2.3 \pm 1.0$ & $1.1 \pm 0.5$ \\
$\begin{array}{l}\text { caudal mucosal } \\
\text { extention }\end{array}$ & $4.4 \pm 1.5$ & $3.6 \pm 1.5$ & $3.8 \pm 1.5$ & $5.8 \pm 1.9$ \\
$\begin{array}{l}\text { mucosal thick- } \\
\text { ness }\end{array}$ & $8.6 \pm 2.5$ & $8.1 \pm 2.3$ & $8.3 \pm 2.5$ & $10.9 \pm 2.9$ \\
projection angle & $57.0 \pm 14.5$ & $63.6 \pm 22.5$ & $83.2 \pm 22.4$ & $84.2 \pm 16.7$ \\
\hline
\end{tabular}

\section{DISCUSSION}

It is a well-known certainty, that the failure rate of isolated septoplasty in cases of nasal obstruction is up to $30 \%{ }^{(7,14,19)}$. Therefore some authors accuse an untreated hypertrophy of the inferior turbinate ${ }^{(9,20)}$. This may be due to the fact that the septum will relocate after surgical correction and can aggravate obstruction of the non-deviated side of the nose. In many patients with a strong anterior septal deviation a compensatory hypertrophy of the inferior turbinate on the concave side of the septum can occur ${ }^{(14)}$. Since the 19th century many surgical techniques have been described for the reduction of the inferior turbinate $^{(15)}$

The reasons for the huge number of surgical techniques are on the one hand the variable anatomy of the inferior turbinate: the hypertrophy can be caused by bony or mucosal changes ${ }^{(8)}$ which have to be treated differently. On the other hand there is no ideal surgical method found, yet ${ }^{(14)}$. There are only limited data in the international literature concerning the surgical anatomy of the turbinates, even though this is a very common issue in clinical routine.

Zuckerkandl (1) within his monograph, as well as Lang ${ }^{(3,4)}$ described the above mentioned anatomic region based on cadaver specimen, respectively. Zuckerkandl (1) stated the mucosal length in anterior-posterior direction with $25-49 \mathrm{~mm}$ and its thickness with $5-16 \mathrm{~mm}$. In the current study the mucosal length was found out to be in all patients $51.0 \pm 5.1 \mathrm{~mm}$ (range $31.3-60.6 \mathrm{~mm}$ ) and its width between $2.5 \mathrm{~mm}$ and $17.2 \mathrm{~mm}$.

Furthermore, the inferior turbinate is described to be often flat and originating from the lateral nasal wall in an acute angle. Otherwise he mentioned it to be sometimes convex, so that he differences an upper horizontal part, which originates in an

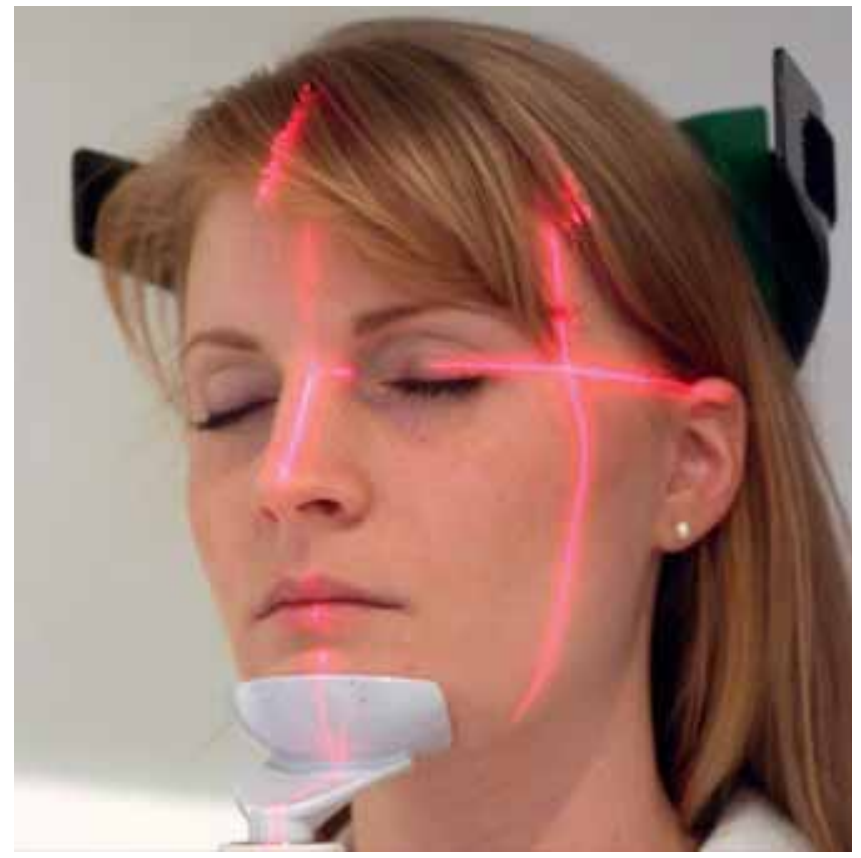

Figure 1. The Region of Interest (ROI) is marked with target beams to define the anatomical examination area. The patient's eyes have to be closed to protect the retina from the laser beams.

orthogonal angle and a downward part with vertical direction. In this study there was also found out to be a great variability in the projection angle. It was $57.0^{\circ}$ at $\mathrm{M} 1,63.6^{\circ}$ at $\mathrm{M} 2,83.2^{\circ}$ at M3 and $84.2^{\circ}$ at M4 (Table 7). The range was between $12.0^{\circ}$ and $163.0^{\circ}$. Additionally, he distinguished the insertion in an anterior, middle and posterior part. Zuckerkandl ${ }^{(1)}$ described the middle one as the longest part with horizontal course which is creating the caudal border of the maxillary ostium. The anterior and posterior parts leave the middle one in an obtuse angle.

In two different studies Lang reported the bony length to be $47.74 \mathrm{~mm}$ (range $35-58 \mathrm{~mm}$ ) ${ }^{(3)}$ and $43.43 \mathrm{~mm}$ (range $35-51$ $\mathrm{mm})^{(3,4)}$. Whereas in the first evaluation he mentioned the right one to be longer than the left one, there were no significant differences between left and right side in the second study. In the current analyses the bony length was detected to be $39.0 \pm 4.0$. To analyse the bony height Lang (3) chose three measuring points. The first is in the rostral section, the second one in the middle section and the last one in the dorsal section. The results were $13.6 \mathrm{~mm}$ (range $6-21 \mathrm{~mm}$ ), $12.9 \mathrm{~mm}$ (range $4-24.5 \mathrm{~mm}$ ) and $10.3 \mathrm{~mm}$ (range $5-17 \mathrm{~mm}$ ), respectively. It is reported that there are no significant differences between male and female patients. In the current study the findings of the bony height were $3.9 \pm 2.0 \mathrm{~mm}$ (range $1.1-9.1 \mathrm{~mm}$ ) at measuring point M1, $20.8 \pm 5.2 \mathrm{~mm}$ (range $5.9-48.5 \mathrm{~mm}$ ) at $\mathrm{M} 2,13.7 \pm 3.1 \mathrm{~mm}$ (range $1.5-31.5 \mathrm{~mm}$ ) at $\mathrm{M} 3$ and $4.0 \pm 2.0$ (range $1.5-16.0 \mathrm{~mm}$ ) at $\mathrm{M} 4$.

Possible reasons for the differences between the results of Zuckerkandl ${ }^{(1)}$ and Lang ${ }^{(3,4)}$ and those of the current study are the choice of different measuring points and the comparison of a cadaveric study with a radiological study.

The differences in the results of the cadaveric analysis of Lang ${ }^{(3,4)}$ and Zuckerkandl ${ }^{(1)}$ may be due to the fact that Lang ${ }^{(3,4)}$ 


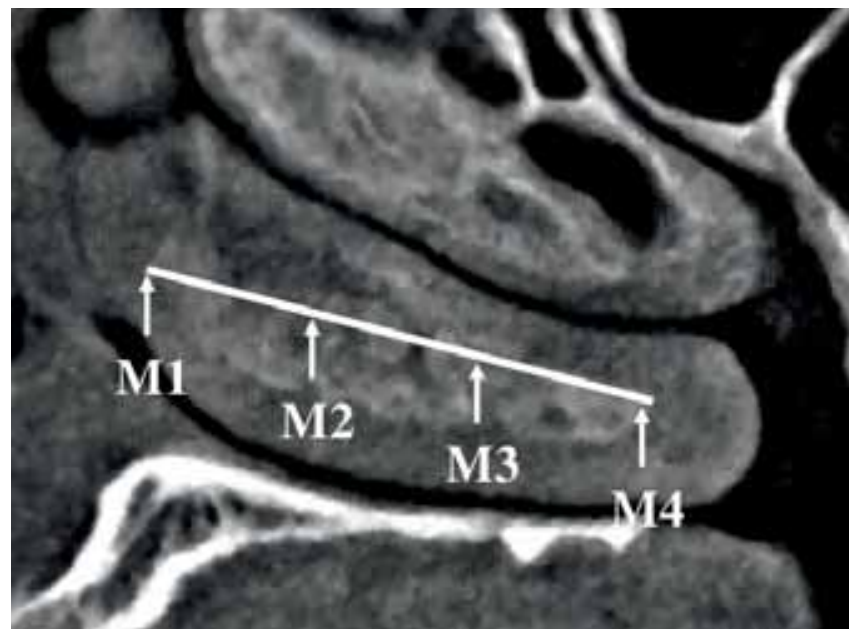

Figure 2. Bony length in anterior-posterior direction with its four measuring points.

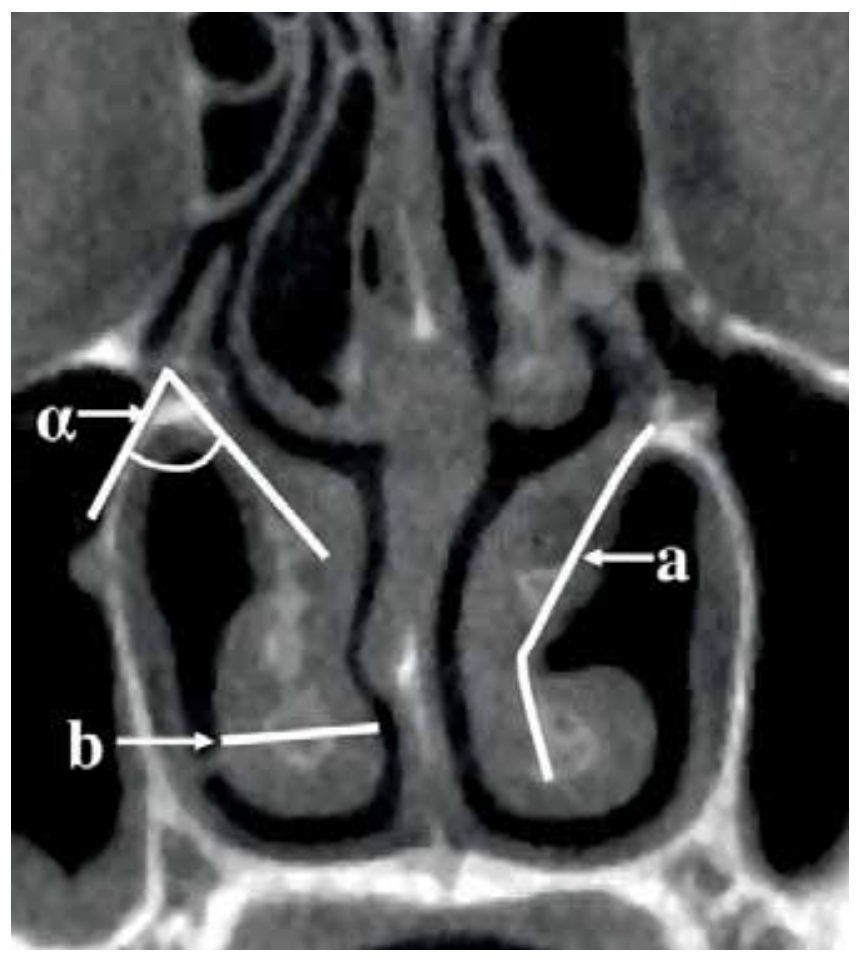

Figure 3. Marking of the bony height (a), the total mucosal thickness (b) and the projection angle $(\alpha)$.

measured the bony height at three measuring points in addition to the bony length. Another important reason for the differences could be the inclusion of patients suffering from nasal symptoms that are suspicious for chronic sinusitis so anatomical changes in the lateral nasal wall can be suspected. Patients with obvious obstructed paranasal sinuses in the DVT scan were excluded from the study, however, to minimise this. On the other hand it is unknown if the individuals examined by Zuckerkandl ${ }^{(1)}$ and Lang ${ }^{(3,4)}$ did not have any nasal complaints during their life.

One weak point in the study population may be the high number of patients with inflammatory symptoms (74 vs. 26 trauma patients) so the values for the mucosal thickness

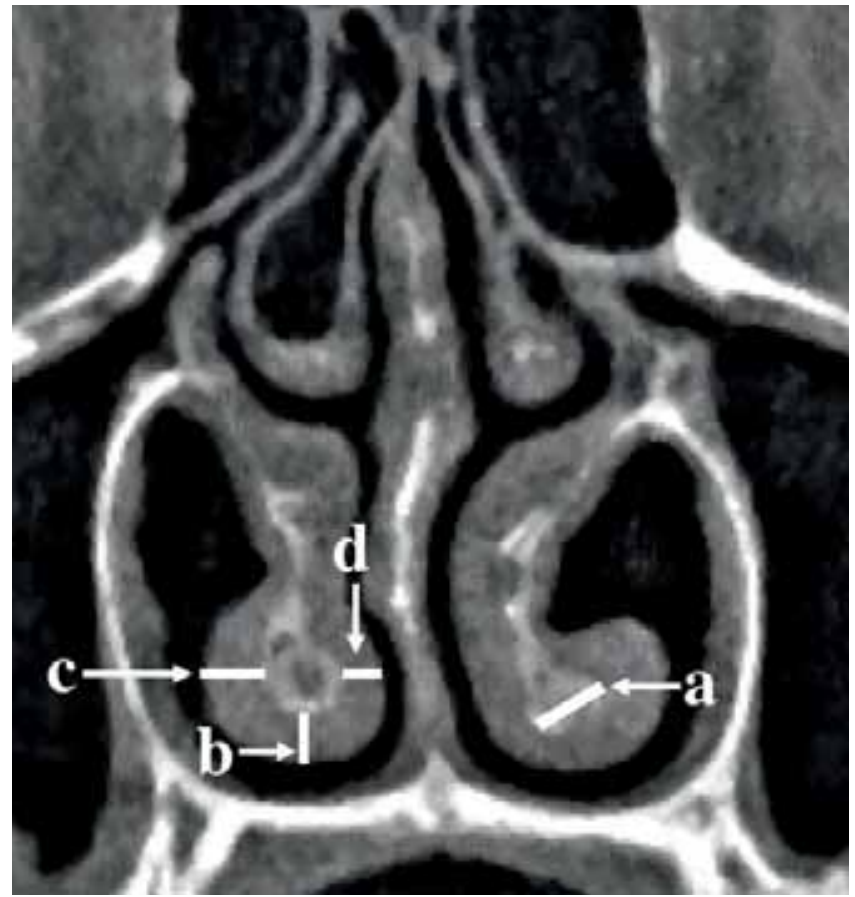

Figure 4. Marking of the bony thickness (a) in its maximal extension, the caudal (b, lateral (c) and medial (d) mucosal thickness.

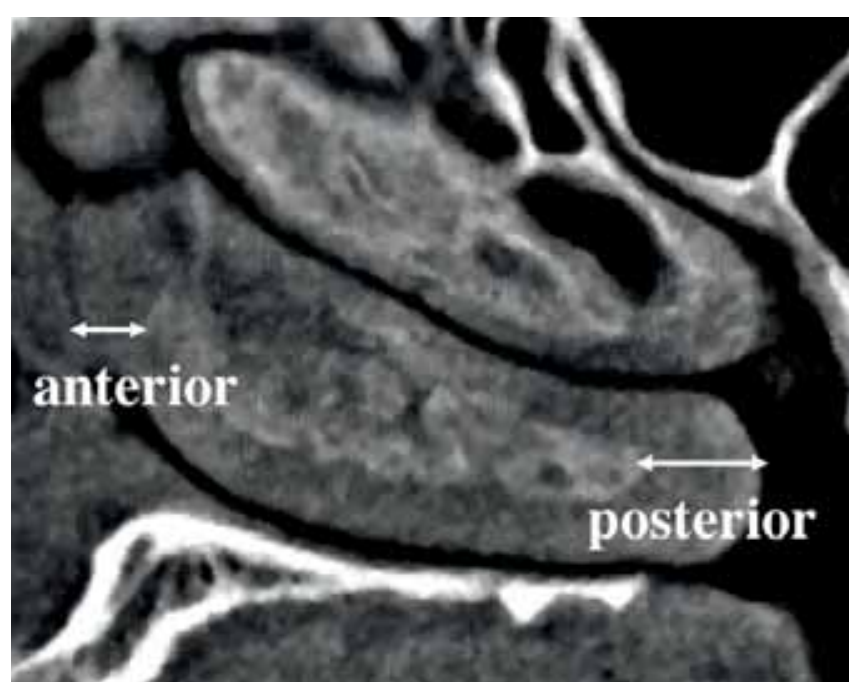

Figure 5. Anterior and posterior mucosal overlay.

may be too high. Another point may be the dimension of the turbinate bone, because it may also be enlarged in cases of chronic inflammation, even though it is unclear if the bony enlargement is one of the reasons for chronic rhinosinusitis or a result of the inflammatory process. Further studies are necessary to examine those theses.

The two subgroups in the current study (patients with trauma and suspected rhinosinusitis) where compared against each other because of the great difference in distribution. This aspect should be also analysed in a further investigation.

It has also to be mentioned that the first diagnostical tool before and after nasal surgery is nasal endoscopy with and without nasal decongestion. The endoscopic results have to be 
supplemented by the radiological finding to get to a substantiated surgical indication.

Today CT scans are the gold standard in paranasal sinus diagnostics. Nevertheless there are little radiologic-anatomical studies of the inferior turbinate, though this knowledge can be very useful preoperatively to choose an adequate treatment concept. The position of $\mathrm{CT}$ as diagnostic tool for radiologic examination of the nasal cavity and the paranasal sinuses is caused by its ability to display microanatomic structures precisely ${ }^{(21)}$. By the use of DVT scans it is possible to evaluate the radiologic anatomy of the turbinates, the nasal cavity and the nasal septum as well in vivo without possible mucosal artefacts caused by fixation of an anatomical specimen. The theoretical resolution which can be achieved by DVT using a slice thickness of $0.08 \mathrm{~mm}$ may be also obtained with the latest generation of CT-scanners but only with much higher financial investments. Current comparative studies concerning this topic do not exist.

DVT is a comparative new radiologic modality for the evaluation of bony pathologies introduced in the 1990's. DVT affords a superior detailed resolution compared to most CT scanners (22) as well as lower radiation exposure in combination with lower costs compared to a 16 row multi slice CT scanner ${ }^{(18)}$.

DVT could demonstrate its ability in the field of ENT in several studies. DVT scans of the midface were evaluated by Bremke and co-workers ${ }^{(18)}$ concerning the display of surgical landmarks of the paranasal sinuses and the anterior skull base. In this study, the authors demonstrated that the DVT volume of $10 \times 10 \mathrm{~cm}$ is able to show all important anatomical landmarks of the anterior skull base as well as the paranasal sinuses ${ }^{(18)}$. In another study, the same group described the DVT as a useful option to conventional radiography in cases of nasal bone fracture because of its specific display of the fracture ${ }^{(16)}$. The radiological anatomy of the olfactory fossa also was successfully evaluated by DVT scans ${ }^{(17)}$.

\section{CONCLUSION}

The results of this radiological study are similar to the ones of cadaveric studies and are able to point out the importance of preoperative anatomical evaluation of the radiological images. The preoperative focus on the individual anatomy is very important because it can give decision support concerning the indication for the best individual surgical concept for the treatment of the hypertrophied inferior turbinate.

\section{ACKNOWLEDGEMENT}

No financial support was received for the realisation of this study.

\section{REFERENCES}

1. Zuckerkandl E. Normale und pathologische Anatomie der Nasenhöhle und ihrer pneumatischen Anhänge. Vol I. ViennaLeipzig: Braunmüller W, 1882.

2. Hilger JA, Hilger PA. Physiology of the nose and paranasal sinuses. In: Goldman JL. Principles and practice of Rhinology. Churchill Livingstone. New York, Edinburgh, London, Melburne, 1987.

3. Lang J, Sakals E. The height of the cavitas nasi, the length of the bony palate and the size and arrangement of the conchae nasales and apertura sinus sphenoidalis. Anat Anz. 1981; 149: 297-318.

4. Lang J, Baumeister R. Postnatal growth of the nasal cavity. Gegenbaurs Morphol Jahrb. 1982; 128: 354-393.

5. Stier, F. Untersuchungen über die Verbiegungen der Nasenscheidewand. Diss, Rostock 1895.

6. Gray L. The deviated nasal septum. I. Aetiology. J Laryng. 1965; 79: 567-755.

7. Grymer LF, Illum P, Hilberg O. Septoplasty and compensatory inferior turbinate hypertrophy: a randomized study evaluated by acoustic rhinometry. J Laryngol Otol. 1993; 107: 413-417.

8. Berger G, Hammel I, Berger R, Avraham S, Ophir D. Histopathology of the inferior turbinate with compensatory hypertrophy in patients with deviated nasal septum. Laryngoscope. 2000; 110: 2100-2105.

9. Polloch RA, Rohrich RJ. Inferior turbinate surgery: an adjunct to successful treatment of nasal obstruction in 408 patients. Plast Reconstr Surg. 1984; 74: 227-236.

10. Fairbanks DNF, Kaliner M. Nonallergic rhinitis and infection ln: Otolaryngology Head and Neck Surgery, 3rd edition Vol. 2. Cummings CW, Fredericson SM, Harker AC, Krause CS, Richardson MA, and Schuller DE (Eds). St. Louis: Mosby, 910920, 1998.

11. Zinreich SJ, Mattos DE, Kennedy DW, Chisholm HL, Diffley DM, Rosenbaum AE. Concha bullosa: CT evaluation. J Comput Assist Tomogr. 1988; 12: 778-784.

12. Bolger WE, Clifford A, Butzin CA, Parsons DS. Paranasal sinus bony anatomic variations and mucosal abnormalities: CT analysis for endoscopic sinus surgery. Laryngoscope. 1991; 101: 56-64.

13. Mirza N, Lanza DC. The nasal airway and obstructing breathing during sleep. Otolaryngol Clin North Am. 1999; 32: 243-262.

14. Lippert BM, Werner AJ. Treatment of hypertrophic inferior turbinate. 1. HNO. 2000; 48: 170-181.

15. Lippert BM, Werner AJ. Treatment of hypertrophic inferior turbinate. 2. HNO. 2000; 48: 267-274.

16. Bremke M, Wiegand S, Sesterhenn AM, Eken M, Bien S, Werner JA. Digital volume tomography in the diagnosis of nasal bone fractures. Rhinology. 2009; 47: 126-131.

17. Savvateeva DM, Güldner C, Murthum T, et al. Digital volume tomography (DVT) measurements of the olfactory cleft and olactory fossa. Acta Otolaryngol. 2009 Nov 2. [Epub ahead of print].

18. Bremke M, Sesterhenn AM, Murthum T, Al Hail A, Al Kadah B, Bien S, Werner JA. Digital volume tomography (DVT) as a diagnostic modality of the anterior skull base. Acta Otolaryngol. 2009; 129: 1106-1114.

19. Uzun L, Savranlar A, Beder LB, Ugur MB, Cinar F, Ozdemir H, Gundogdu S. Enlargement of the bone component in different parts of the compensatory hypertrophied inferior turbinate. Am J Rhinol. 2004; 18: 405-410.

20. Feder RJ, Partial turbinectomy. Laryngoscope. 1984; 94: 259-260.

21. Leunig A, Betz CS, Sommer B, Sommer F. Anatomic variations of the sinuses; multiplanar CT-analysis in 641 patients. Laryngorhinologie. 2008; 87: 482-489.

22. Mozzo P, Procacci C, Tacconi A, Martini PT, Andreis IA, A new volumetric CT machine for dental imaging based on the conebeam technique: preliminary results. Eur Radiol. 1998; 8: 15581564 .

Prof. Dr. Jochen A. Werner

Department of Otorhinolaryngology

Head and Neck Surgery

Marburg, UKGM

Baldingerstrasse 3

35043 Marburg

Germany

E-mail: wernerj@med.uni-marburg.de 